\title{
Performance analysis on color image mosaicing techniques on FPGA
}

\author{
Jayalaxmi H ${ }^{1}$, S. Ramachandran ${ }^{2}$ \\ ${ }^{1}$ Department of Electronics \& Communication Engineering, Acharya Institute of Technology, India \\ ${ }^{2}$ Department of Electronics \& Communication Engineering, SJBIT, India
}

\section{Article Info \\ Article history: \\ Received Jun 11, 2018 \\ Revised Dec 3, 2018 \\ Keywords: \\ CIM \\ DCT \\ Device utilization \\ Execution time \\ FPGA \\ Image mosaicing \\ MSE \\ PSNR \\ SWM}

Accepted Dec 15, 2018

\section{Corresponding Author:}

Jayalaxmi H,

Department of Electronics \& Communication Engineering,

Acharya Institute of Technology, Bengaluru, India.

Email: jayalaxmiresearchwork@gmail.com

\begin{abstract}
Today, the surveillance systems and other monitoring systems are considering the capturing of image sequences in a single frame. The captured images can be combined to get the mosaiced image or combined image sequence. But the captured image may have quality issues like brightness issue, alignment issue (correlation issue), resolution issue, manual image registration issue etc. The existing technique like cross correlation can offer better image mosaicing but faces brightness issue in mosaicing. Thus, this paper introduces two different methods for mosaicing i.e., (a) Sliding Window Module (SWM) based Color Image Mosaicing (CIM) and (b) Discrete Cosine Transform (DCT) based CIM on Field Programmable Gate Array (FPGA). The SWM based CIM adopted for corner detection of two images and perform the automatic image registration while DCT based CIM aligns both the local as well as global alignment of images by using phase correlation approach. Finally, these two methods performances are analyzed by comparing with parameters like PSNR, MSE, device utilization and execution time. From the analysis it is concluded that the DCT based CIM can offers significant results than SWM based CIM.
\end{abstract}

Copyright (C) 2019 Institute of Advanced Engineering and Science. All rights reserved.

\section{INTRODUCTION}

The customized advancement of high-resolution image mosaics is a dynamic research domain ofcomputer graphics, vision and image processing [1]. The image mosaicing is normally used to fabricate the visual view field by sticking together various video sequences. The camera's view field is always less than human view field [2]. Also, large objects often can't be caught in a single image as in aerial photography. Using a convergence lens for broader view is a kind of solution but it will have some sort of distortion in capturing the image. Also, the capturing the entire scene with less resolution may also induce image quality issues [3]. The panoramic image mosaics can be created by using some devices like Video Recording, surround video thatmoves around the camera optical core interest [1]. Also, it has strong imperatives on the imaging conditions. Thus, to overcome this, image alignment, image saucing and image frames pasting can be used to get complete view of image [4].

The minimum complex mosaics are produced by using an image sets whose mutual displacement is a pure image place translation. This is the approximate case which takes place in satellite images translation. The image translation can either be handled by physically demonstrating corresponding centers or by an image correlation [5]. Other direct mosaics are generated by rotating the camera at its optical concentration by a device and generating a panoramic image that indicates the scene projection over a cylinder. But is quite tough task to have clear image under different light intensity, noise, orientation, alignment etc [5]. 
The minimum complex mosaics are produced by using an image sets whose mutual displacement is a pure image place translation. This is the approximate case which takes place in satellite images translation. The image translation can either be handled by physically demonstrating corresponding centers or by an image correlation [5]. Other direct mosaics are generated by rotating the camera at its optical concentration by a device and generating a panoramic image that indicates the scene projection over a cylinder. But is quite tough task to have clear image under different light intensity, noise, orientation, alignment etc [5].

This section evolves with the discussion on existing works on image mosaicing techniques. The image mosaicing is a concept of combining two or more image and forms asingle image without any visible seam lines. A survey work on image mosaicing is performed in Ghosh and Kaabouch [6] that discuss existing image mosaicing algorithms, classification of mosaicing technique, state of art in current research trend etc. In order to construct the mosaiced image various algorithms and techniques were introduced [7]. Most of the research methods have employed significant point matching techniques and corner detection models [8]. An interesting work of Zagrouba et al. [9] have introduced Harris points primitives [10] and regions based image mosaicing concept which helps to improve the mosaicing performance against the issues like illumination variation, brightness, noise etc. In the work of Zhu and Ren [11] Scale Invariant Feature Transform (SIFT) [12] based image mosaicing method is illustrated. Primarily, the method uses operator from Harris corner detection [13] and detects the key points. Then constructs directed line segments for rough point matching. The final outcomes of the method suggest its robustness against rotation, scaling, resolution and lighting issues. The work of Elibol et al. [14] has introduced underwater image mosaicing by using submapping. This approach utilizes a modified agglomerative clustering (hierarchical) mechanism to form the submaps based on similarity among the feature matching among the image which reduces computational cost. The real time image mosaicing is introduced in Kekec et al. [15] for aerial images. By Jaziri [16] the algorithm has been developed by using a low-cost FPGA. This research study also presented a capable design methodology which provides benefits of significant design. The major benefits show the re-configurable hardware modules for the electrical methods. The outcomes showed the model platform that presents the effectiveness and the profits of the proposed viewpoint. Rajendran and Devarajan [17] have developed a new approach to design of a high-performance torque control technique. This study also focused on direct torque control with space vector modulation of three phase introduction motor by using FPGA. Ismael et al. [18] have presented a view point to present the Bresenham algorithm byusing separating every line into number of sections. The outcome shows the maximum number of segments presented, and points are calculated. In this axis theorem is utilized to identify the image intersection and affine refinement mechanism to extract exact global consistency. The final outcomes of the method give the promising results in obtaining the better results of mosaicing. From the review analysis it is found that very rare researches were considered the alignment issues, image quality and resolution issues, manual registration and also very less real time implementation is performed for mosaicing. The following section gives the issues which are considered in this research.

Recently, most of the techniques were offered to build the mosaiced image under homography variations in the image. If the overlapping among the two images is high mosaicing may give good results (through Levenberg Marquardt approach [19]) but yields sensitive against local minima and causes higher computational complexity. Similarly, if the overlapping among the two images is low, the hierarchical matching can bring significant results to avoid local minima. For the images with improper alignment with cross correlation approach and faces issues with image brightness variation. Also, very rare researches were observed for image mosaicing with hardware implementation to cover the image overlapping. The mosaiced images need to be aligned properly with proper mosaicing algorithms. This paper considers the correlation problem or alignment problem, image quality and resolution issues during image mosaicing. Also, the manual image registration and real-time implementation of mosaicing algorithm is considered. In that sense, two different approaches of image mosaicing are introduced which implements Sliding Window Module and Discrete Cosine Transform separately to perform automatic image registration and alignment issues respectively.

\section{PROPOSED IMAGE MOSAICUNG SYSTEM}

This section gives the design of (a) SWM based colour image mosaicing approach for corner detection to perform automatic image registration (b) DCT based color image mosaicing to tackle image alignment issues.

\subsection{SWM based CIM}

This kind of image mosaicing approach considers two color images ( $1 \& 2)$ and parallelly both the images will be subjected to SWM technique. Each color images will have red (r), green (g), and blue (b) 
components and for these components are subjected with SWM individually. The SWM technique consists of image preprocessing module and it is used to create its neighboring pixels and forming the $3 \times 3$ window. Once the window is formed convolution method is applied using Gaussian filtering method. The convolution method includes both the horizontal and vertical matrix components. After that gradient module collects the gradients from convoluted image and corner detection is performed. Once the corner detection is completed for the images, the $\mathrm{r}, \mathrm{g}, \mathrm{b}$ components of both the images will be integrated to form the mosaiced image. The architecture of SWM based CIM is given in Figure 1.

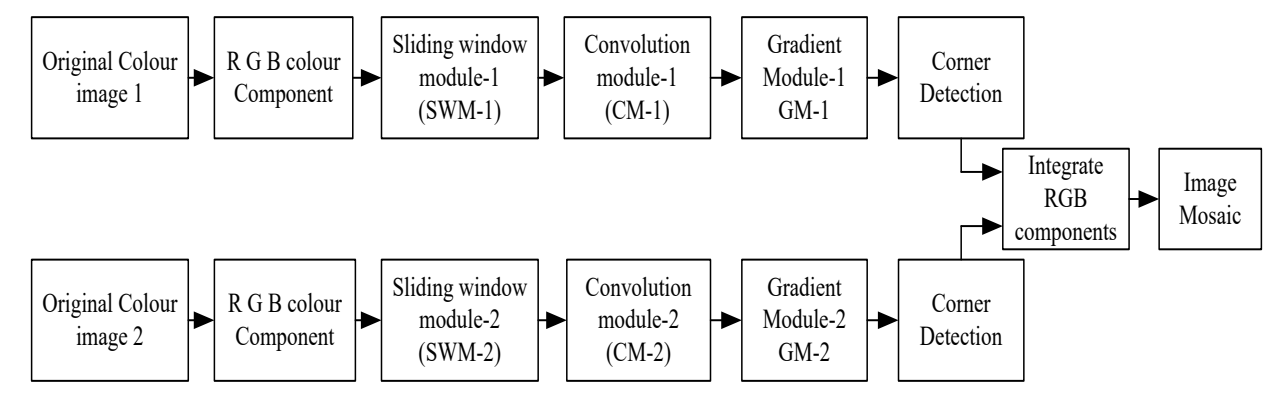

Figure 1. Novel architecture for the sliding window based image mosaicing

The following Figure 2 shows the internal architecture of the CIM using SWM. The architecture contains six Main Sliding Window (MSW) and three individual corner detection model to generate componentsr, g, and $\mathrm{b}$ of mosaiced color image. Here, the 8-bit $\mathrm{r}, \mathrm{g}, \mathrm{b}$ components of both the images (1 and 2) are subjected to MSW module. Further, the outputs of r, g, and b components from MSW module will be given to corner detection (corner $\mathrm{r}$, corner $\mathrm{g}$ and corner $\mathrm{b}$ ) module as $\mathrm{r}, \mathrm{g}, \mathrm{b}$ components separately. The corner matching helps to take a small pixel's region as a window from the detected corner and compare it for same region witheverycorner features in the other image. The extracted corners from each window are fed to the Image transformation module. Two sets of corner values in the images have been detected using corner module. The convoluted derivatives are fed as input to the next module to compute each image corner values from the two images being mosaiced. The choice of corner for feature detection is stable when corner is greater than 1. Once the two sets of corner values in the images have been detected, the aim is to match the corresponding features to align the images.

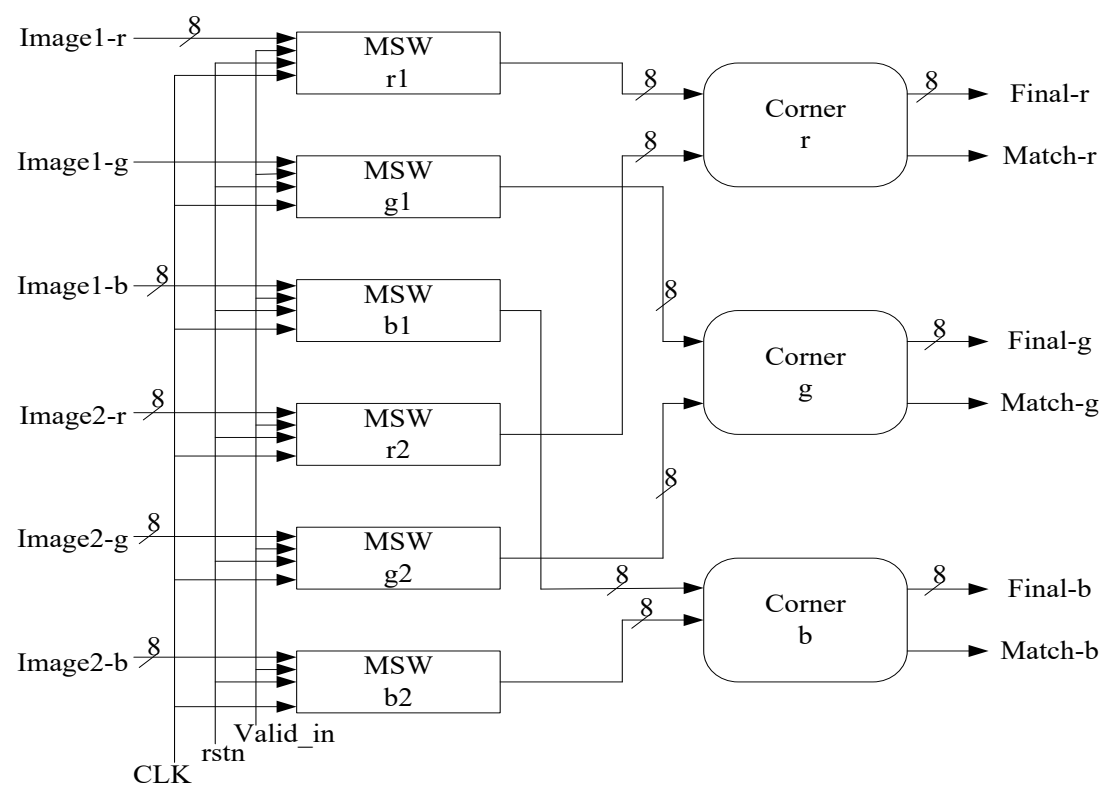

Figure 2. Internal architecture of CIM using SWM 


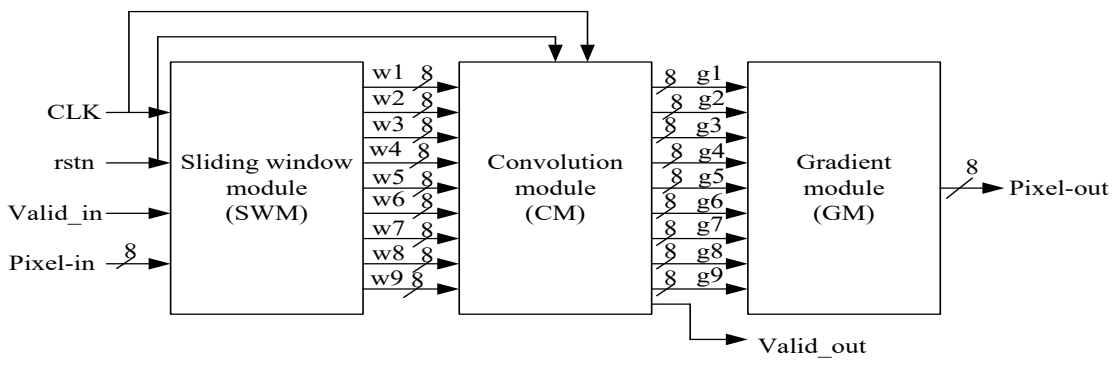

Figure 3. Internal Structure of MainSliding window (MSW) module

The internal structure of MSW module is shown in Figure 3, which consists of three modules like SWM, Convolution Module (CM) and Gradient Module (GM). Once the clock is activated, an asynchronous reset signal will be high along with valid_signal=1 (high). The 8-bit pixel_in is the input of the color of any of the rgb components. The 8-bit pixel_in input is coming serially based on the clocks and it will store in the memory locations. The stored pixels create its neighboring pixels to form a SWM module. Each window module is having $3 \times 3$ matrixes; hence nine different 8 bit window modules (W1-W9) are generated. These nine different window modules are convoluted using Gaussian filtering with horizontal and vertical matrix. Finally, it generates gradients (g1 to g9). The gradient module collects the all the nine gradient inputs to sum up and truncate with last 8-bit values to generate final 8-bit pixel value.

\subsection{DCT based CIM}

The DCT based CIM approach aims to tackle the local and global alignment issues by using phase correlation technique. The following Figure 4 indicates the block diagram of the proposed DCT based CIM. This model of CIM also considers two color images (1 and 2) and extracts the RGB components from them. These RGB components are feed to DCT engine as input. Then DCT engine is performed over two images where the images will be parted in different frequencies. The DCT engine is used to perform cosine transformation using memory coefficients. The DCT will perform compressed image output using 2D-DCT algorithm. Then it will be forwarded to multiplier and divider module. The DCT output composed of 2D-DCT's which is input to multiplier which generate product of two multipliers. The output of multiplier is given to divider, which divide the rgb components of image. The main use of multiplier and divider is used to find the similar overlapped pixels of two images. The DCT approach helps to resolve most of the overlapped image parts of two images. Later, Then IDCT engine is applied for phase correlation is calculated. From that image registration is done and then registration values are generated. Image registration values are considered as a final mosaicing image. The image registrations process helps to check the aligned pixels in corners of two images. If the aligned pixels are same then only the registration will take place otherwise the pixels are not aligned. This process takes place till the last pixels of two images.

The above Figure 5, represents the DCT based CIM top module that composed different input 8 bit components like red (r), green (g) and blue (b) of image 1 and 2, clock (CLK), reset (rst and Start) and respective outputs (dout_r, dout_g, dout_b. done_r, done_g and done_b). Figure 6 shows internal structures of multiplier and divider.

The above Figure 7, indicates the Inverse DCT's internal architecture that composes various blocks like shift register, adder/substractor, complement check, multiplier block, adder and updation of output in RAM memory. Here, 8-bit memory coefficient is chosen to store the 1D-DCT constant coefficient value for multiplication operation. Further on the basis of counter index, the coefficients are allotted to 8-bit memory. These 8 bit input data will be shifted to 8 times by using shift registers. These shifted 8-bit inputs will be registered in each $8^{\text {th }}$ block. On the basis of toggling, addition or subtraction is shifted to output values of 8 bit. Further addition or subtraction is subjected for the shifted 8bit output values. The output of MSB bit will further analyze the addition and subtraction output. Later, multiplication operation is performed where the memory 1, 2,3,4,5,6,7,8 with adder/subtractor outputs can yield the 8-different products p1, p2...p7. Finally, all the products will be added to generate final output of adder. The generated adder output is rounded off to get the output coefficient (1D-DCT). The transpose of memory taken to get the immediate coefficients that stores the 64 coefficients in 64 location of RAM memory. Later, on the basis of read/write signals the RAM module is enabled by using the counter. The first DCT generated coefficient will be appeared in RAM output. At next, the process of 2D-DCT is begins to generates its corresponding output by repeating the above process. 


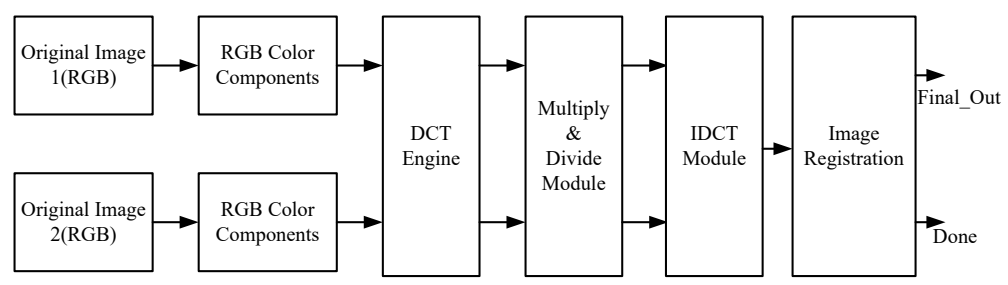

Figure 4. Block model of DCT based CIM

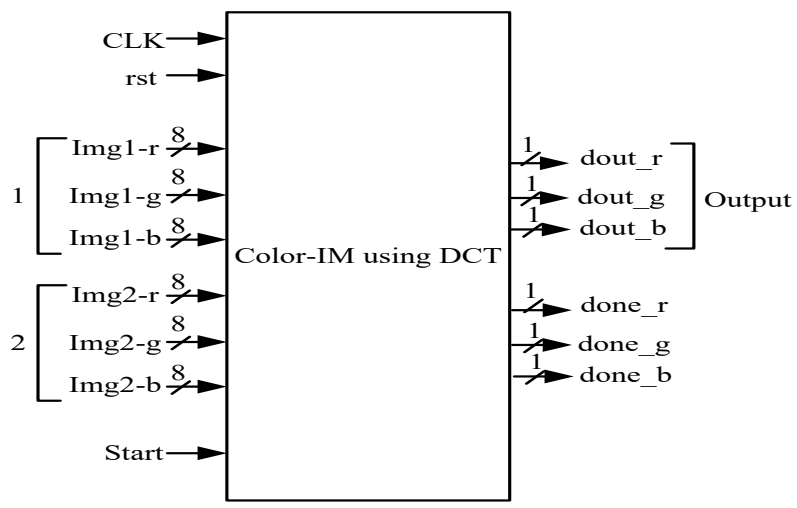

Figure 5. Top module of DCT based colour image mosaicing system

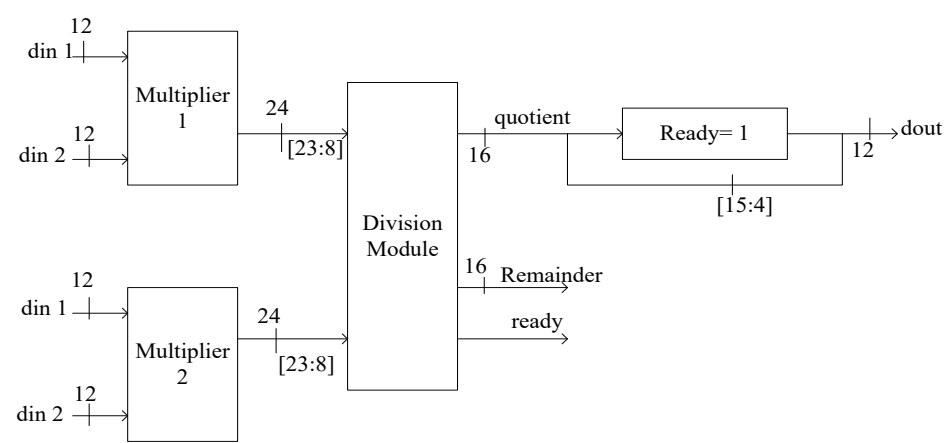

Figure 6. Internal structures of multiplier and divider

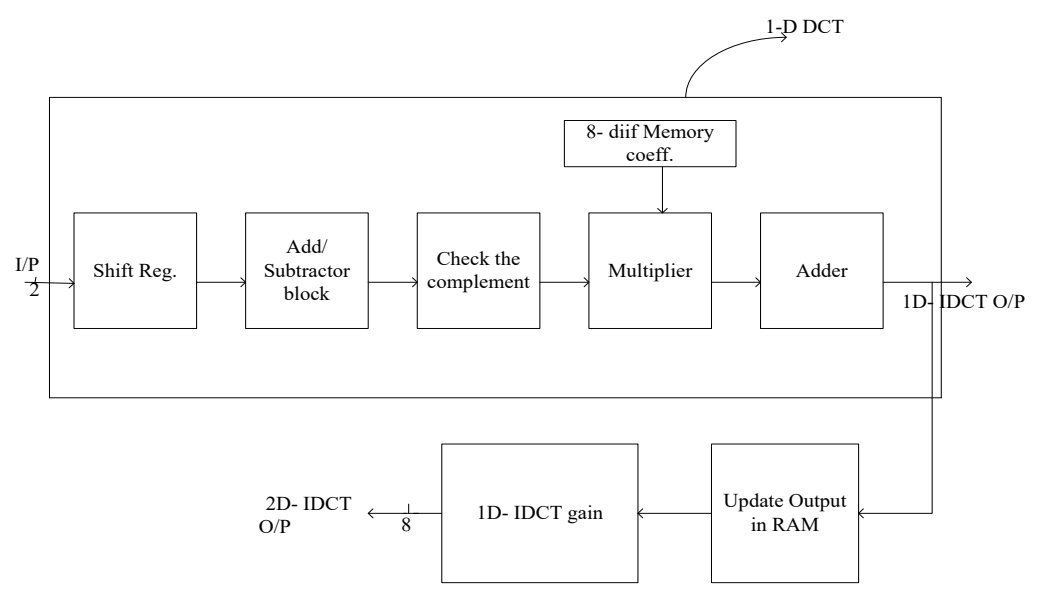

Figure 7. Internal architecture of inverse DCT 


\section{RESULTS AND DISCUSSIONS}

Both the CIM systems using SWM and DCT approaches are designed using Verilog coding. Further the validation of these designs is performed by considering appropriate tools that supports the design. Hence, the system execution is performed in Xilinx 14.7 ISE and simulation using Modelsim 6.3f. The complete design is held over Artix-7 FPGA board of device 7A100T-3 CSG324. Following are the obtained results after successful execution of the system.

The above Figure 8 indicates the CIM outcomes obtained for pepper image of size 1600x1200 using SWM and DCT approaches. In this, left image and right image used to create mosaiced image by matching its features. Further, the comparative analysis of these two approaches SWM and DCT is performed for parameters like PSNR, MSE, device utilization and execution time. The following table gives the corresponding comparison values of PSNR, MSE, device utilization and execution time in Tables 1-4 respectively.

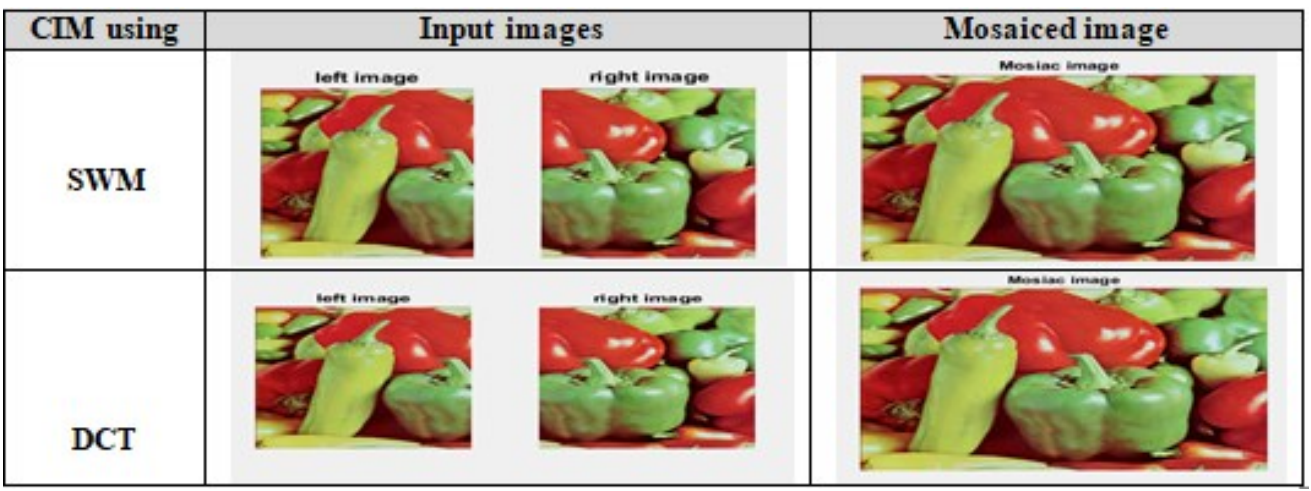

Figure 8. CIM for 1600x1200 peppers image

Table 1. PSNR Comparison of SWM and DCT based CIM

\begin{tabular}{cc}
\hline Parameter/Method (Peppers.jpg) & PSNR value in \\
\hline CIM using SWM & $31.6844 \mathrm{~dB}$ \\
CIM using DCT & $36.2882 \mathrm{~dB}$ \\
\hline
\end{tabular}

Table 2. MSE Comparison of SWM and DCT based CIM

\begin{tabular}{cc}
\hline Parameter/Method (Peppers.jpg) & MSE value \\
\hline CIM using SWM & 43.0884 \\
CIM using DCT & 15.2849 \\
\hline
\end{tabular}

Table 3. Device Utilization

\begin{tabular}{llll}
\hline \multicolumn{1}{c}{ Device Utilization } & Available & SWM based CIM & DCT based CIM \\
\hline Number of Slice Registers & 126800 & 116121 & 66988 \\
Number of Slice LUTs & 63400 & 252660 & 94612 \\
Number of completely used LUT-FF pairs & 97127 & 115521 & 64473 \\
Number of bonded IOBs & 210 & 79 & 70 \\
Number of BUFG/BUFGCTRLs & 32 & 9 & 2 \\
\hline
\end{tabular}

Table 4. Execution Time Comparison of SWM and DCT based CIM

\begin{tabular}{ccc}
\hline Approach & CIM using SWM & CIM using DCT \\
\hline Execution time & $38.42 \mathrm{~ms}$ & $23.02 \mathrm{~ms}$ \\
\hline
\end{tabular}

\section{CONCLUSION}

This paper presented two different image mosaicing methods like a) SWM based color image mosaicing (CIM) approach for corner detection to perform automatic image registration, (b) DCT based color image mosaicing to tackle image alignment issues. Both the methods considered the two images as inputs to form mosaiced image. A comparative analysis is performed among both the methods. By analyzing the tables of PSNR, MSE, device utilization and execution time of SWM and DCT based CIM, it is found that effective PSNR value of DCT $(36.2882 \mathrm{~dB})$ than SWM (31.6844 dB), least MSE value of DCT (15.2849) than SWM (43.0884), less device utilization of DCT than SWM and it takes low execution time of DCT (23.02ms) than SWM (38.42ms). The higher value of PSNR indicates the good quality of mosaiced image. Least MSE value represents the low error in mosaiced image and utilizes less hardware components. This paper can be implemented for surveillance system to monitor the abnormal actions. The proposed methods can be considered in future researches to have further improvement in performance. 


\section{REFERENCES}

[1] Szeliski, Richard, and Heung-Yeung Shum., "Creating full view panoramic image mosaics and environment maps", Proceedings of the 24th annual conference on Computer graphics and interactive techniques. ACM Press/AddisonWesley Publishing Co., 1997.

[2] Reinhard, Erik, et al., "High dynamic range imaging: acquisition, display, and image-based lighting," Morgan Kaufmann, 2010.

[3] Stern, Adrian, and Bahram Javidi., "Three-dimensional image sensing, visualization, and processing using integral imaging," Proceedings of the IEEE 94.3: 591-607, 2006.

[4] Bhosle, Udhav, Subhasis Chaudhuri, and Sumantra Dutta Roy., "A fast method for image mosaicing using geometric hashing," IETE Journal of Research 48.3-4: 317-324, 2002.

[5] Murino, Vittorio, and Andrea Trucco., "Three-dimensional image generation and processing in underwater acoustic vision," Proceedings of the IEEE 88.12: 1903-1948, 2003.

[6] Ghosh, Debabrata, and Naima Kaabouch., "A survey on image mosaicing techniques," Journal of Visual Communication and Image Representation 34: 1-11, 2016.

[7] Zitova, Barbara, and Jan Flusser., "Image registration methods: a survey," Image and Vision Computing 21.11: 977-1000, 2003

[8] Garcia-Fidalgo, E., Ortiz, A., Bonnin-Pascual, F. and Company, J.P., "A mosaicing approach for vessel visual inspection using a micro-aerial vehicle," In Intelligent Robots and Systems (IROS), IEEE/RSJ International Conference on (pp. 104-110), IEEE, September- 2015.

[9] Zagrouba, Ezzeddine, Walid Barhoumi and Slim Amri., "An efficient image-mosaicing method based on multifeature matching," Machine Vision and Applications 20: 139-162, 2007.

[10] Abraham, Rintu, and Philomina Simon., "Review on mosaicing techniques in image processing," Advanced Computing and Communication Technologies (ACCT), 2013 Third International Conference, IEEE, 2013.

[11] Zhu, Jun, and Mingwu Ren., "Image mosaic method based on SIFT features of line segment," Computational and mathematical methods in medicine, 2014.

[12] Chen, Shuai, et al., "Image mosaic based on SIFT and morphological component analysis," Image and Signal Processing, BioMedical Engineering and Informatics (CISP-BMEI), 10th International Congress, IEEE, 2017.

[13] Amaricai, Alexandru, Constantina-Elena Gavriliu, and Oana Boncalo., "An FPGA sliding window-based architecture harris corner detector," Field Programmable Logic and Applications (FPL), 24th International Conference. IEEE, 2014.

[14] Elibol, Armagan, et al., "Fast Underwater Image Mosaicing through Submapping," Journal of Intelligent \& Robotic Systems 85.1: 167-187, 2017.

[15] Kekec, Taygun, Alper Yildirim, and Mustafa Unel., "A new approach to real-time mosaicing of aerial images," Robotics and Autonomous Systems 62.12: 1755-1767, 2014.

[16] Jaziri, Ibtihel. "A Simplified Speed Control of Induction Motor based on a Low Cost FPGA," International Journal of Electrical and Computer Engineering (IJECE) Vol 7, no. 4, 1760-1769, 2017.

[17] ]Rajendran, R., and N. Devarajan. "Simulation and implementation of a high performance torque control scheme of IM utilizing FPGA," International Journal of Electrical and Computer Engineering (IJECE) Vol 2, no. 3, 277-284, 2012.

[18] Ismael, S, O. Tareq, Y.T. Qassim, "Hardware/Software Co-design for a Parallel Three-Dimensional Bresenham's Algorithm," International Journal of Electrical and Computer Engineering (IJECE), 9, no.1, Retrieved on 16-Oct, 2018

[19] Szeliski, Richard, "Image mosaicing for tele-reality applications," Applications of Computer Vision, Proceedings of the Second IEEE Workshop, IEEE, 1994.

\section{BIOGRAPHIES OF AUTHORS}

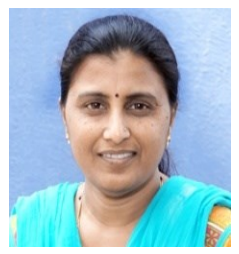

Jayalaxmi $\mathbf{H}$ received the M.Tech degree in VLSI and Embedded System from BMSCE, Bangalore, VTU, India, in 2005. She is currently working in the department of ECE, Acharya Institute of Technology, Bangalore, India and pursuing PhD in JNTU, Hyderabad. Her current research interests include image/video processing, VLSI and published papers in IEEE, Elsevier Science Direct, and ACEEE.

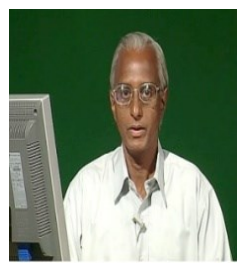

S. Ramachandran, having a Ph. D. in EE/VLSI Design from IIT Madras. He is currently a Professor in SJB Research Foundation, SJBIT, Bangalore, establishing Hi Tech Research Labs and guiding a number of Research scholars. He has written a book titled Digital VLSI Systems Design published by Springer Verlag, Netherlands, which is followed as a text book for BS/MS/Ph. D. in many Universities of USA, Europe and other parts of the World. Also, a Video Course has been completed at IIT Madras on Digital VLSI System Design for NPTEL, National telecast on TV. 